\title{
GASO 2-2015: MASGULINO DE 86 AÑOS CON MASA INTRA ABDOMINAL: TUMOR DEL ESTROMA GASTROINTESTINAL
}

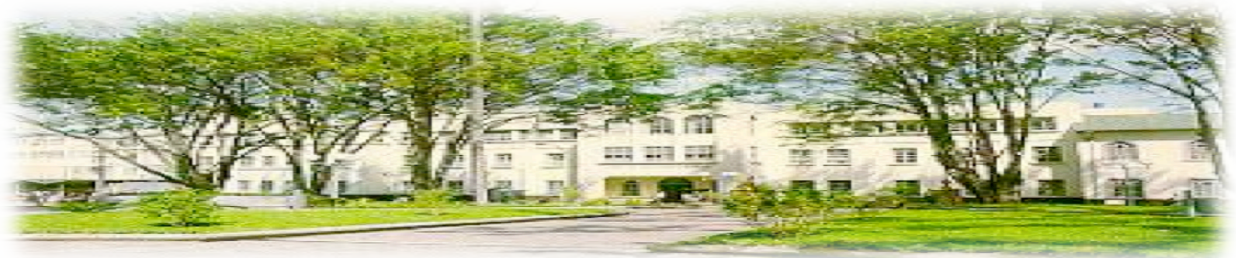

Hospital San quan de Dios. San José. Costa Rica. Fundado en 1845

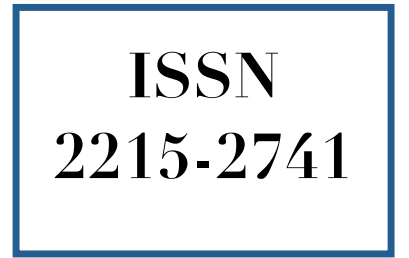

Irene Mora Quesada ${ }^{1}$

Carlos Barrantes León ${ }^{2}$

\footnotetext{
${ }^{1}$ Médica General. Universidad de Costa Rica. Correo electrónico: ire.moraq@gmail.com

${ }^{2}$ Médico Asistente Especialista en Cirugía General. Hospital Max Peralta. C.C.S.S. Cartago Costa Rica. Correo electrónico: barranleon@hotmail.com
}

\section{RESUMEN}

Masculino de 86 años de edad, hipertenso, con enfermedad pulmonar obstructiva crónica, antecedente de hiperplasia prostática benigna y masa intra abdominal en estudio. Consultó por cuadro de dolor abdominal con hallazgos clínicos y de laboratorio compatibles con síndrome de lisis tumoral. Fue llevado a sala de operaciones donde se realizó resección tumoral, con posterior diagnóstico histopatológico de tumor del estroma gastrointestinal (TEGI).

\section{PALABRAS CLAVE}

Tumor del estroma gastrointestinal. Proto oncogen c-kit. Factor de crecimiento derivado de plaquetas. Inhibidores de tirosina kinasa.

\section{ABSTRACT}

The case of an 86 years old male patient, with medical history of hypertension, chronic obstructive pulmonary disease, benign prostatic hyperplasia and intra abdominal mass is presented. $\mathrm{He}$ came to the E.R. complaining of abdominal pain, with clinical and laboratory findings consistent with tumor lysis syndrome, for that reason is brought to the O.R., underwent tumor resection and after, histopathological diagnosis of GIST was made.

\section{KEY WORDS}

Gastrointestinal stromal tumor. Proto oncogene c-kit. Platelet derived growth factor. Tyrosine kinase inhibitors. 


\section{CASO CLÍNICO}

Paciente masculino de 86 años, vecino de Cartago, con antecedentes médicos de hipertensión arterial, enfermedad pulmonar obstructiva crónica e hiperplasia prostática benigna. Con historia de masa abdominal en estudio, como hallazgo incidental de un ultrasonido control realizado en febrero de 2014, en control en el hospital Max Peralta Jiménez, con intervención quirúrgica programada para Julio de 2014.

Se presentó al servicio de emergencias médicas en julio de 2014 con cuadro de dolor abdominal intenso, difuso, persistente, de horas de evolución asociado a sensación febril sin otros síntomas. En la exploración física se documentó diaforético, taquicárdico, taquipneico, febril, con piel marmórea, distensión abdominal, con dolor a la palpación en fosa iliaca derecha y masa palpable. No se auscultó ruidos peristálticos.

Se realizó ultrasonido (US) de abdomen el cual demostró una masa intraabdominal sólida, heterogénea, con focos de calcificación, de 9,7 x $7,8 \times 6 \mathrm{~cm}$. Además una tomografía de abdomen, con hallazgo de masa en fosa ilíaca derecha, de $10 \mathrm{~cm}$ de diámetro, sólida, homogénea, que desplazaba las asas intestinales sin generar obstrucción, que impresionaba ser de origen extra colónico, con escaso líquido libre en la cavidad abdominal.

Los estudios diagnósticos realizados en fechas anteriores a dicho internamiento corresponden a dos colonoscopias fallidas, un tránsito intestinal sin alteraciones estructurales, una radiografía de tórax con hallazgo de lesiones nodulares en ambos campos pulmonares.

Tomografía de abdomen de abril 2014 que documentó masa en fosa ilíaca derecha, de contornos definidos, hipodensa, con algunas áreas líquidas en su interior, de $7 \times 8.2 \times 9.3 \mathrm{~cm}$, en contacto y generando compresión extrínseca de las asas intestinales circundantes, sin poder descartar origen intestinal.

No se reportó adenopatías ni lesiones hepáticas. Además, un US de abdomen en junio de 2014 describió una formación redondeada de $100 \times 80$ x $112 \mathrm{~mm}$ en fosa ilíaca derecha, de bordes definidos, vascularizada, sin líquido libre en cavidad abdominal.

El paciente fue llevado a sala de operaciones con diagnóstico de aparente síndrome de lisis tumoral. Se realizó laparotomía exploratoria con hallazgo de masa sangrante de $10 \mathrm{~cm}$ de diámetro, localizada en el borde anti mesentérico de íleon, a $40 \mathrm{~cm}$ de la válvula ileocecal, adherida a la serosa del sigmoides y la vejiga, con escasa cantidad de sangre en fosa ilíaca derecha.

Se realizó resección segmentaria del íleon de 10 $\mathrm{cm}$, con entero-entero anastomosis latero-lateral mecánica, con rafia de la pared vesical y disección de la masa adherida a la pared del sigmoides sin complicaciones.

Fue egresado en el día número ocho posterior a la intervención, en buen estado general, sin complicaciones asociadas al procedimiento.

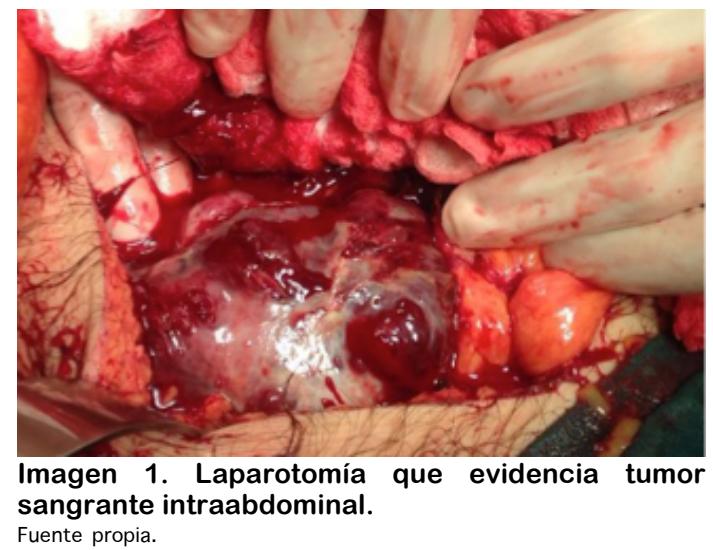

El diagnóstico patológico demostró un tumor del estroma gastrointestinal, de $110 \mathrm{~mm}$ de diámetro, con rango mitótico de 2 en CAP, clasificado como de alto riesgo, con necrosis de $30 \%$ del tumor, inmunohistoquimica positiva para CD117, S-100, CD 34, actina de músculo liso. Márgenes negativos.

El paciente continúa en control en cirugía general del hospital Max Peralta Jiménez. 


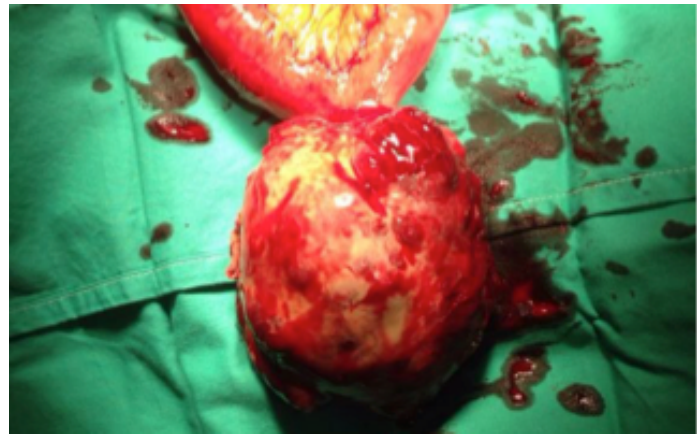

Imagen 2. Tumor del estroma gastrointestinal en el borde anti mesentérico de íleon.

Fuente propia.

\section{DISCUSIÓN Y ANÁLISIS DEL CASO}

Los tumores del estroma gastrointestinal (TEGI) son neoplasias derivadas de las células intersticiales de Cajal o de sus precursores celulares ${ }^{(1-3)}$. Representan entre $0.1 \%$ al $3 \%$ de todas las neoplasias gastrointestinales y equivalen aproximadamente al $80 \%$ de los tumores del mesénquima gastrointestinal $^{(1,4,5)}$. Aproximadamente el $80 \%$ presentan mutaciones en el proto oncogen c-kit, mientras que en 5 a $8 \%$ de los casos se presentan mutaciones en el receptor $\alpha$ del factor de crecimiento derivado de plaquetas, PDGFRA por sus siglas en inglés ${ }^{(1-3)}$.

Existen tres subtipos de TEGI basados en su composición histológica, $70 \%$ presentan células en huso, $20 \%$ células epiteloides y mixtas en $10 \%^{(1,3-5)}$. El primero consiste en fascículos celulares uniformes que se intersecan, mientras los dos subtipos restantes corresponden a células redondeadas con núcleos atípicos organizadas en forma laminar o anidada ${ }^{(2)}$.

Se presentan con mayor frecuencia en adultos entre los 40 a 80 años, sin preferencia de sexo o etnia $^{(1,3,5)}$. La mayoría de los casos son sintomáticos, sin embargo hasta $20 \%$ se diagnostican de forma incidental, principalmente tumores que miden $<3 \mathrm{~cm}^{(1,2,5)}$. La distención y el dolor abdominal asociados a masa palpable intra abdominal corresponden a los principales motivos de consulta. Algunos casos se presentan con obstrucción intestinal, sangrado intra abdominal y/o anemia $^{(1,2,5)}$.

El tamaño medio tumoral al momento del diagnóstico en pacientes sintomáticos es de $5 \mathrm{~cm}^{(2)}$.
Hasta $60-70 \%$ de los casos se encuentran localizados a nivel gástrico, 20 a $25 \%$ en intestino delgado, $5 \%$ colorectal y $5 \%$ en esófago ${ }^{(1)}$.

Los TEGI se presentan de forma infrecuente asociados a otros tumores, formando parte de síndromes clínicos como en el caso de CarneyStratakis, triada de Carney, Neurofibromatosis tipo 1 y TEGI familiar ${ }^{(3,4,6)}$.

La enfermedad metastásica se presenta al momento del diagnóstico hasta en $50 \%$ de los pacientes, localizada principalmente a nivel de hígado y peritoneo ${ }^{(3,4)}$. Las metástasis extra abdominales son poco frecuentes, los sitios principales de afectación corresponden a pulmones, pleura, hueso, tejido subcutáneo y sistema nervioso central ${ }^{(2,4)}$. Es importante destacar que al ser tumores derivados del estroma gastrointestinal, solo en raras ocasiones involucran ganglios linfáticos, por lo que su resección no es necesa$\mathrm{ria}^{(1)}$.

Los métodos diagnósticos incluyen estudios de imágenes, como resonancia magnética o tomografía computarizada de tórax, abdomen y pelvis $^{(1,5)}$. Típicamente se observan como masas con crecimiento extrínseco extra luminal, con crecimiento expansivo no infiltrativo que desplaza las estructuras vecinas ${ }^{(1,2)}$. Otros estudios como la tomografía emisora de positrones resultan útiles al ser tumores metabólicamente activos y se utilizan particularmente en la evaluación de la respuesta al tratamiento ${ }^{(1,5,6)}$.

No se recomienda realizar estudios histológicos previos a la cirugía, debido a la posibilidad de diseminación tumoral, sin embargo, en los casos en los que se considere necesario, se puede llevar a cabo una biopsia endoscópica o percutánea $^{(1,2,5)}$.

El diagnóstico definitivo se obtiene mediante inmunohistoquímica. Se utilizan marcadores para kit (antígeno CD117); positivo en 95\% de los casos, DOG1; positivo en $95 \%$ de los casos, el CD34; positivo en $70 \%$ de los casos, actina de músculo liso; positiva en $30-40 \%$ de los casos, S$100 ; 5 \%$, desmina $2 \%$ y citoqueratina $2 \%{ }^{(3,5,6)}$.

La caracterización molecular tiene gran importancia tanto para el diagnóstico como para el tratamiento. Se ha determinado que la mayoría 
de las mutaciones de c-Kit, localizadas en el brazo largo del cromosoma 4, se encuentran en el exón 11 y presentan mejor respuesta a tratamiento farmacológico, mientras que las mutaciones en el exón 9, las cuales se presentan hasta $10 \%$ de los casos, se asocian con menor respuesta al tratamiento farmacológico, por lo que requieren dosis más altas ${ }^{(1,4)}$.

Los tumores que presentan mutaciones en PDGFRA localizados principalmente en el estómago, presentan en su mayoría adecuada respuesta al tratamiento, sin embargo, la mutación más frecuente, localizada en el exón 18 , es resistente a la terapia farmacológica ${ }^{(1,2,4)}$.

Carney-Stratakis, triada de Carney y Neurofibromatosis tipo 1 forman parte de los tumores de tipo silvestre, los cuales representan aproximadamente el 10\%; denominados de esta manera porque no presentan mutaciones en c-kit o PDGFRA $^{(3,6)}$.

El tamaño, la actividad mitótica y la localización anatómica son los criterios utilizados para estratificar el riesgo de malignidad, el potencial metastásico y el riesgo de recurrencia, siendo el conteo de figuras mitóticas el principal determinante de riesgo independiente ${ }^{(1,3,5,6)}$. Existen distintos nomogramas en los que se han utilizado otros factores pronósticos como la ruptura tumoral, con el objetivo de evaluar mejor el riesgo individual $^{(3,5,6)}$. Los tumores pequeños $(<2 \mathrm{~cm})$, con baja actividad mitótica $(<5$ en 50 CAP) se asocian con bajo potencial maligno mientras que aquellos de mayor tamaño $(>5 \mathrm{~cm})$, con mayor actividad mitótica ( $\geq 5$ en $50 \mathrm{CAP}$ ) se asocian más frecuentemente con comportamiento maligno y mayores tasas de recurrencia post quirúrgica $^{(2)}$.

El único tratamiento curativo es la resección quirúrgica, lo que es cierto para tumores primarios y localizados, los cuales representan el $60 \%$ de los casos ${ }^{(2,5-8)}$. El tratamiento de elección para los tumores $\geq$ a $2 \mathrm{~cm}$ de diámetro es la resección quirúrgica, sin embargo el tratamiento para los tumores más pequeños con bajo riesgo de malignidad ha sido controversial. Se recomienda actualmente mantenerlos en vigilancia endoscópica cada 6 a 12 meses, siempre y cuando estos no presenten sintomatología y/o aumenten de tamaño ${ }^{(1,2,5-9)}$.
En el caso de tumores $<2 \mathrm{~cm}$ localizados en sitios de mayor potencial maligno, como el intestino delgado y el colon, se recomienda la resección quirúrgica, basado en su riesgo de sangrado o metástasis ${ }^{(9,10)}$.

Durante la cirugía se debe explorar el abdomen en busca de metástasis peritoneales y/o hepáti$\operatorname{cas}^{(7)}$. La resección quirúrgica con márgenes microscópicos negativos es el tratamiento con mejores resultados a largo plazo, sin embargo la extensión ideal de los márgenes se desconoce ${ }^{(1,5)}$. La meta del cirujano es lograr lo anterior sin rotura tumoral, manteniendo la pseudo cápsula tumoral intacta ${ }^{(2,6,7)}$. Algunos cirujanos recomiendan realizar resección peritoneal local con la finalidad de eliminar las siembras tumorales a este nivel ${ }^{(7)}$.

En tumores gástricos es aceptable la obtención de márgenes microscópicos de varios milímetros, lo que se puede obtener con la resección en cuña, principalmente en tumores localizados en la curvatura mayor $^{(1)}$. En tumores de mayor tamaño se realiza gastrectomía segmentaria o total ${ }^{(1)}$.

En caso de tumores pequeños de intestino delgado la resección segmentaria con márgenes macroscópicos de 2-3 cm es de elección, sin embargo se pueden realizar desde resecciones en cuña, que presentan mayores tasas de recurrencia que la resección segmentaria, hasta pancreaticoduodenectomia, que presenta tasas de mortalidad y sobrevivencia similares a las resecciones segmentarias, pero mayor morbimortalidad asociada al procedimiento ${ }^{(1,7)}$. Sin embargo en tumores periampulares o de $>3 \mathrm{~cm}$ localizados en la primera o segunda porción del duodeno, está indicada la realización de pancreaticoduodenectomia $^{(7)}$.

En los tumores localizados en el colon está indicada la resección segmentaria ${ }^{(7)}$. A nivel rectal los tumores pequeños pueden ser resecados por vía tras anal o tras esfínter, mientras que en tumores de mayor tamaño la resección puede ser tras sacra, tras vaginal o abdomino perineal ${ }^{(7)}$. Sin embargo, esta zona anatómica presenta una alta tasa de márgenes positivos asociada a la mayor dificultad técnica ${ }^{(7)}$.

En los casos en los cuales se realizan resecciones R1 una nueva cirugía puede llevarse a cabo, sin 
embargo no existen datos estadísticos que demuestren diferencias en la supervivencia libre de enfermedad entre resecciones R0 y R1, por lo tanto esta no se recomienda ${ }^{(5,6,8)}$.

Actualmente se recomienda la cito reducción pre quirúrgica con inhibidores de actividad kinasa de la tirosina-kinasa, con el objetivo de obtener márgenes quirúrgicos óptimos y disminuir el riesgo de metástasis en los casos en los que sea difícil realizar resecciones R0, además en casos considerados de alto riesgo de sangrado o ruptura intraoperatoria $^{(1,6)}$. Sin embargo la duración de la terapia y la adecuada selección de los pacientes continúan en estudio ${ }^{(2,5,6,8)}$.

El imatinib, un inhibidor de tirosina kinasa (ITK) es la terapia de primera línea para enfermedad metastásica o recurrencia ${ }^{(3,5,6)}$. Sin embargo es importante tomar en cuenta que aproximadamente el $50 \%$ de los pacientes desarrolla resistencia luego de 2 años de tratamiento. En estos casos está aprobado el uso de un segundo inhibidor de tirosina kinasa ${ }^{(3)}$. El uso de terapia adyuvante con ITK ha demostrado ser efectiva en pacientes con tumores de riesgo intermedio o alto, mejorando la sobrevivencia libre de enfermedad, la sobrevivencia global y disminuyendo la tasa de recu$\operatorname{rrencia}^{(2,5,6)}$.

Las recurrencias son principalmente abdominales, involucran hígado en $66 \%$ de los casos y peritoneo en 33\%. Las recurrencias en los márgenes de resección son poco frecuentes y usualmente son irresecables ${ }^{(1,2)}$. No obstante en los casos en los que una nueva resección quirúrgica es posible, es de elección la combinación con tratamiento farmacológico ${ }^{(2)}$. En los casos de enfermedad metastásica avanzada, la reducción de la masa tumoral mediante cirugía podría prolongar el tiempo previo a que se genere resistencia al tratamiento farmacológico ${ }^{(6)}$.

El seguimiento con estudios de imagen está indicado en todos los casos. Los tumores de bajo riesgo pueden recurrir con metástasis varios años después de la cirugía por lo tanto se debe dar seguimiento a largo plazo, se recomienda realizar estudios de imágenes de abdomen y pelvis cada 6 a 12 meses por 5 años y posteriormente cada año ${ }^{(1,8)}$. Mientras que en tumores de medio y alto potencial maligno se recomienda cada 3 a 6 meses por 3 años, cada 6 meses hasta los 5 años y posteriormente control anual ${ }^{(1,8)}$.

\section{CONCLUSIONES}

Los tumores del estroma gastrointestinal representan una baja proporción de los tumores gastrointestinales, sin embargo el estudio continuo de esta patología ha permitido que su conocimiento continúe evolucionando.

La implementación de la inmunohistoquimica $\mathrm{y}$ la caracterización molecular han permitido mejorar el diagnóstico, la estratificación de riesgo y la adecuada selección de pacientes para terapia farmacológica.

El advenimiento de la terapia con inhibidores de tirosina kinasa ha revolucionado el tratamiento y pronóstico de los pacientes con TEGI, sin embargo son necesarios otros estudios clínicos para determinar la duración ideal del tratamiento y otras interrogantes con respecto a esta terapia.

La cirugía sigue siendo el tratamiento de elección y debe ser llevada a cabo siempre que sea posible.

\section{BIBLIOGRAFÍA}

1. Eisemberg B Pipas JM. Gastrointestinal Stromal Tumor - Background, Pathology, Treatment. Hematol Oncol Clin N Am. 2012,;26:1239-1259.

2. Bamboat Z Dematteo R. Updates on the Management of Gastrointestinal Stromal Tumors. Surg Oncol Clin N Am. 2012;21: 301-316.

3. Goldblum J Folpe A Weiss S. Enzinger and Weiss's Soft Tissue Tumors. Elsevier. 6 Edition, 2014.

4. O`Regan K Shinagare A Saboo S Ramaiya $\mathrm{N}$ Jagannathan J Tirumani S. Gastrointestinal stromal tumors (GIST): lesser known facts. Clinical Imaging. 2013;37:821-829.

5. Cameron J Cameron A. Current Surgical Therapy. Elsevier. $11^{\text {th }}$ Edition, 2014.

6. Joensuu $\mathrm{H}$ Hohenberger $\mathrm{P}$ Corless $\mathrm{C}$. Gastrointestinal stromal tumour. Lancet. 2013;382:973-983. 
7. Fernández J Sánchez $M$ Parrilla $P$. Controversies in the surgical treatment of primary gastrointestinal stromal tumours. Cir Esp. 2010;88:69-80.

8. Casali P Blay J. Gastrointestinal stromal tumours: ESMO Clinical Practice Guidelines for diagnosis, treatment and follow-up. Annals of Oncology. 2010:21 (S5):98-102.

9. Tetsuji F. Management of small gastrointestinal stromal tumours (Correspondence). Lancet. 2013;382:1701.

10. Joensuu H Hohenberger P. Management of small gastrointestinal stromal tumours (authors reply). Lancet. 2013;382:1701-1702.

\section{CONFLICTO DE INTERÉS}

Los autores declaran que no existió ningún conflicto de interés en el presente reporte. 